\title{
Excuse Me, Do I Know You From Somewhere? Unaware Facial Recognition Using Brain-Computer Interfaces
}

\author{
Christopher Bellman \\ University of Ontario Institute \\ of Technology \\ Christopher.Bellman@uoit.net
}

Ruba Alomari

University of Ontario Institute of Technology

Ruba.Alomari@uoit.ca

\author{
Miguel Vargas Martin \\ University of Ontario Institute \\ of Technology \\ Miguel.VargasMartin@uoit.ca
}

\author{
Shane MacDonald \\ University of Ontario Institute \\ of Technology \\ Shane.MacDonald2@uoit.net
}

\author{
Ramiro Liscano \\ University of Ontario Institute of Technology \\ rliscano@ieee.org
}

\begin{abstract}
While a great deal of research has been done on the human brain's reaction to seeing faces and reaction to recognition of these faces, the unaware recognition of faces is an area where further research can be conducted and contributed to. We performed $a$ preliminary experiment where participants viewed images of faces of individuals while we recorded their EEG signals using a consumer-grade BCI headset. Pre-selection of the images used in each of the three phases in the experiment allowed us to tag each image based on what state of recognition we expect the image to take - No Recognition, a Possible Unaware Recognition, and a Possible Aware Recognition. We find, after filtering, artifact removal, and analysis of the participants' EEG signals recorded from a consumer-grade BCI headset, obvious differences between the three classes of recognition (as defined above) and, more specifically, unaware recognitions, can be easily identified.
\end{abstract}

\section{Introduction}

Facial recognition research is not a newly studied field and it has been the focus of many studies over the past number of decades (see Section 2 - Related Work). While conscious facial recognition is becoming more and more understood and studied, facial recognitions at an unaware level is an area that has potential to be investigated, especially with advancements in modern technologies that allow us to approach the research from different angles.

Electroencephalography (EEG) is a technology that uses electrodes placed on (non-invasive) or in/under (invasive) the skin, skull, or even the brain to record the brain's electrical activity [1]. While traditional EEG setups are most commonly used and associated with the medical and neuroscience research community, Brain-Computer Interfaces (BCIs) have been used in a more general and non-medical manner to record these EEG signals without having to resort to a medical procedure or lab setup. In recent years, noninvasive consumer-grade BCI headsets have been made available to the market at reasonable prices to allow for cheaper, broader access to EEG technology. While modern consumer-grade headsets do not have the features or quality that the medical-grade EEG caps have, they are a useful tool for consumers and researchers to be able to work with, play with, and study the human brain. Some examples of consumer use for BCI devices include EMOTIV's wide variety of brain-based games, which include such activities as controlling an RC helicopter [2] or playing a game of Tetris [3], all with your brain. Other examples include NeuroSky's online store where you can find games such as bowling [4], or even a BCI version of the widely popular Flappy Bird game [5]. With rapid advancement in modern EEG technologies and the introduction of consumer-grade non-invasive BCI headsets, it seems to suggest that EEG signals and BCI devices may start to become more and more involved in our daily lives, perhaps someday being able to accurately control your music based on your mood, or drive your car by thinking about it. With these potential practical application on the horizon, we, in this work, show how we can utilize the human brain to be able to recognize faces that we are unaware that we have seen previously. We define an "unaware recognition" as a participant "recognizing a face without being aware that any facial recognition took place in their mind". 
One of the brain's features we can use to analyze the brain's response to stimuli are Event-Related Potentials (ERPs). These ERPs are the brain's reaction to stimuli and appear as positive and negative spikes or changes in voltage in the brain's EEG signal, and are simply ranges of time after a stimuli that we expect these spikes to occur [6]. There are a number of ERPs that are commonly used when analyzing EEG signals, and we are focusing on a few specific ones most commonly related to facial recognitions (see Section 2 - Related Work), as well as a more general look at the signal to determine any apparent differences between our three classifications of recognition; No Recognition (NR), Possible Unaware Recognition (PUR), and Possible Aware Recognition (PAR).

We designed an experiment where participants wearing BCI headsets are shown a series of images containing faces of individuals, and are told to view the images and consider them. We break the experiment up into three phases - simply "Phase One", "Phase Two", and "Phase Three" - where the participants are shown these faces, but with specific images being shown in different orders and at varying times. As the participants view these images, we record their EEG signals which are later analyzed to determine if unaware facial recognitions can be accurately recorded and classified using BCI devices and modern machine learning and analysis techniques.

\section{Related Work}

EEG analysis using BCIs to gather data has become a very useful tool for gaining insight into the workings of the human brain and how it can be used to interact with computer systems. This relatively cheap and safe technology works as an alternative for those without the means to take part in more expensive, elaborate, and invasive brain-reading. From computer security [7] to visual design [8], BCIs are being used in a number of fields to enhance and improve current techniques, methodologies, and to add new understanding in the way our brains work and react.

In the next few sections, we discuss previous works regarding such topics as unaware ERP elicitations and application, facial recognitions, and EEG signals and their processing and analysis.

\subsection{Subconscious ERP Elicitations}

While work regarding subconscious recognitions using EEGs and BCIs are few, a number of works have been done regarding the subconscious and the brain's subconscious reaction to events or activities. A number of authors use "subconscious" in their work but we prefer to use the term "unaware" for describing events that take place in the brain without awareness.

Vargas Martin et al. [9] performed an experiment where images of faces of famous individuals were shown to participants in the hopes that roughly $20 \%$ of images shown would be conscious recognized by the participants and $80 \%$ would not. Being that famous people are more likely to be seen often in passing without conscious recognition, it was assumed that the participants' subconscious would recognize a subset of the faces in the $80 \%$ group. They used different ERPs for the testing and training of a support vector machine (SVM) and found that they were able to determine, with about $65 \%$ accuracy across all explored ERPs, which faces in the set of famous individuals were subconsciously recognized. Our work presented here is similar to Vargas Martin et al.'s experiment as faces were used as targets for aware and unaware recognitions, but famous individuals were used and the assumption was based on participants recognizing some, but not all of the individuals presented to them in their paper. We use faces that are assumed to not be recognized at all and use a twophase approach to add an unaware recognition of certain faces which are learned in the first phase. This helps to remove any assumptions on participants' levels of recognition of famous individuals. We hope that with our analysis we can provide further depth into the brain's reaction to different classes of recognition.

Shalgi and Deouell [10] ran an experiment studying error processing in the human brain with regards to conscious or unconscious errors where they had participants bet money (real) on whether or not they could answer questions in the experiment session correctly. The level of wager that the participants placed on an answer was used to define a level of confidence in the answer. They found that an ErrorRelated Negativity index (ERN, an index for error processing in the brain) remained the same for both conscious and unconscious scenarios, but when only looking at high-confidence trials (where participants bet a larger sum of money), the ERN was only noticed for errors that the participant was conscious of (i.e., they were aware of the error). This led them to conclude that ERNs are related to awareness of an error and that the amplitude of the elicited signal is related to confidence.

The Implicit Association Test (IAT) [11] is a test to determine if a participant has any associations (both conscious and unconscious) between characteristics such as human ones (e.g. gender, physical characteristics, religion). Upon taking the IAT, a participant may find biases or associations that they 
were unaware of along with associations that they had (e.g. dark vs. bright symbolizing good vs. evil, being a more obvious and common association). This understanding that the brain can unconsciously think and associate without the conscious mind being aware of it is interesting and allows us to delve further into the workings of the brain with respect to facial processing and recognition.

To our knowledge, little other work has been published regarding the subconscious recognition of things or faces using EEG signals and consumer-grade BCI devices.

\subsection{Facial Recognition}

Facial recognition is a process that human beings do from dozens to potentially thousands of times per day, and plays a huge role in our person-to-person communication. From judging the structure of the face, to the analysis of facial emotions or features, the brain's perception of faces is crucial in our recognition process.

According to Seeck et al. [12], it was believed human visual analysis did not take place before the first $100 \mathrm{~ms}$ after visual stimulus, but they challenged these assumptions. They had participants view faces and recorded their EEG signals and found two Visual Evoked Potentials (VEPs) - one occurring at the 50$90 \mathrm{~ms}$ interval, and then again at the 190-600 ms, thus challenging the previous notion that visual analysis does not take place earlier than $100 \mathrm{~ms}$ [12]. George et al. [13] confirmed the findings of Seeck et al. by finding VEPs at the $50 \mathrm{~ms}$ range for facial recognition.

To challenge these findings by Seeck and George, Debruille et al. [14] made the claim that it is facial repetition - not recognition - that triggers VEPs before the $100 \mathrm{~ms}$ mark, and that facial recognition takes place in the post-100 ms time period. To back up these claims, Eimer [15] ran a study regarding facial recognition and found that facial identification happens in the 130-200 ms range (the "N170" ERP), which takes place separately from, and does not affect, the facial recognition and familiarity process (confirming Bentin and Deouell's study [16]). Along with this finding of facial identification, it was found that the facial recognition and familiarity processes take place in the 300-500 ms range ("N400f" ERP) and the 500-700 ms range ("P600f" ERP) [15]. While Eimer's experiment [15] asked the participants to judge their level of familiarity with a face shown to them (chosen via results of a pilot study), our study makes use of faces that are assumed to be completely unfamiliar to the participants and faces are implicitly learned during the study. Participants of our study are not asked to do any action to indicate their level of recognition during the later phases of the experiment, thus minimizing artifacts or bias from any movement or activity. With this knowledge of recognition, varying levels of recognition were judged. In a study by Bentin et al. [16], participants were shown faces of famous individuals and non-famous, unfamiliar individuals, and found that all faces shown triggered an N170 ERP (confirming Eimer's findings [15]), but faces of individuals that were recognized (famous individuals, in this case) elicited greater amplitude during the N400 ERP whereas unfamiliar faces elicited lesser amplitudes [16]. To supplement these results, Caharel et al. [17] found that in a facial recognition study of famous faces, non-famous (unfamiliar) faces, and faces of the participant, less familiar faces exhibited larger positive amplitudes whereas self-images of the participant exhibited smaller amplitudes.

The Sternberg task [18] tests and measures a participant's response time when presented with a stimulus (in the case of Sternberg's experiment, symbols rather than images of faces), and it was found that a positive recognition had a more rapid response time than a negative recognition by an average of 50 ms. These findings may assist in our work as aware facial recognitions may result in EEG signals with lesser amplitudes than those that are unaware recognitions, or even faces that are not recognized at all, along with a knowledge that a recognized face may show signals earlier than an unknown face to a subject.

Research by Mnatsakanian et al. [19] has suggested that certain ERPs exist specifically for facial recognition and processing (e.g. N170 [15] [16], VEPs [12] [13]), which differs from non-facial recognition that may be associated with different ERPs. This insight offers us a suggestion of which specific ERPs to focus on when considering facial recognition tasks, and assists in future work on the subject.

\subsection{EEG and Processing}

A number of studies have taken place using BCIs and EEG data to further understand the brain's function when assigned a variety of jobs or actions. In a study by Solovey et al. [20], participants were given a variety of multi-tasking activities. They used Functional Near-Infrared Spectroscopy (fNIRS) [21] to analyze the participants' brains during the tasks and were able to determine the mental processes involved in multi-tasking [20]. Peck et al. [8] also made use of fNIRS in analyzing the brain's perception of visual designs. fNIRS data was recorded from participants viewing designs and choices within them and they found they were able to determine how the brain reacts to visual design [8]. 
There are examples of research done using machine learning techniques to classify various features from EEG signals such as Lee et al. [22], who used a Bayesian Network classifier on EEG data from an experiment which tasked participants with a variety of cognitive and non-cognitive activities. They found that they could classify and identify the various tasks performed by the participants with high average accuracies of $\sim 93 \%$ [22]. Our work does not make use of machine learning techniques as the signals are easily differentiated using a basic threshold, but in future works with greater number of participants, machine learning may be required to classify the different levels of recognition within the signals.

The actual processing of EEG signals has been the subject of study for a long time, and still seems to be under debate as to which methods provide the best results, especially in the BCI community. There are various toolsets and libraries dedicated to assisting in the capture, processing, manipulation, and analysis of EEG signals from BCIs. For our work here, we choose to use EEGLAB [23], a MATLAB toolbox, for manipulation and pre-processing of raw EEG signals, then we provide our own analysis and post-processing.

\section{Experiment}

In order to determine the feasibility of classifying unaware facial recognitions using BCIs, an experiment was conducted with a total of three phases. In the first phase, a number of images depicting unknown (to the participant) faces were shown to participants. In the second phase, the same number of images were shown to participants, but a number of images from the first phase were included in this second phase to serve as possible unaware recognitions. The rest of the images in this phase were unknown faces, much like all of phase one. In the final phase - phase three - the same number of images were shown again to the participants, but this time the participants were given a single face from the list of unknown faces pulled from phase one and added to phase two, and were asked to memorize the face and continue when they felt comfortable that they had the face memorized. Additional images were also taken from phase one and added in to phase three to improve the number of possible unknown recognitions that each participant had. In this preliminary study, the three phases were conducted separately across two days (phase one on day one, phases two and three on day two) to prevent possible conscious recognition of images intended to be subconsciously recognized.

At all times during the experiment while participants are viewing images (or the gaps in between), their brains' electrical output is being measured (EEG) and recorded using the EPOC headset, which is then used as the input for our analysis. No manipulation or analysis is done during the experiment - all data is saved until after phase three for processing and analysis.

\subsection{Participants}

For this preliminary study, the participants only included two of the five authors of this paper. Since there is no deception involved in the experiment and the images chosen for each phase of the experiment are unknown via random selection based on a pre-defined seed, the research team can take part in the experiment with minimal bias, however, this does not mean that there is no impact on the study. The research team is aware of the structure of the experiment which may alter the way the individuals' brains react to seeing the images of different classifications (No Recognition, Possible Unaware Recognition, and Possible Aware Recognition). This is a limitation that we hope to address in future work exploring the results of this study where we will use a broader general participant pool with minimal knowledge of the experimental setup and design.

\subsection{Experimental Setup and Stimuli}

All phases of the experiment used the Emotiv EPOC headset [24] for data capture, which records/samples data from the brain at $128 \mathrm{~Hz}$, producing 14 channels (each sensor) of readings taken approximately every $7.8125 \mathrm{~ms}$. Each of these channels can be reconstructed to form graphs of the signals similar to those depicted in Figures 1, 2, 3, and 4.

The EPOC BCI device is a consumer-grade EEG headset. We are interested in using this headset due to its potential ubiquity among consumers who may own BCI headsets for entertainment or accessibility purposes, rather than lab technicians and medical research personnel. This ubiquity may allow for more rapid realization of practical applications in a more meaningful and consumer-friendly way than a large, expensive, and complicated BCI headset. While we would not consider the EPOC as a toy that someone would normally pick up at a toy store, we do consider it a toy in general seeing that many games and interactive applications have been developed for entertainment purposes [3]. The EPOC headset has 14 electrodes, all located according to the 10-20 system for EEG electrode positioning [25]. The 14 electrodes used on the headset are: AF3, F7, F3, FC5, T7, P7, O1, O2, P8, T8, FC6, F4, F8, and AF4. They are placed 
externally on the scalp using pre-dampened felt pads to conduct the signal through the skin. Due to this, the EPOC is considered a non-invasive type of BCI [1].

For this experiment, all images of faces shown to participants come from the FERET database [26] [27]. These images are of individuals' faces positioned in the center of the image, converted from colour to grayscale, and only images that are frontal shots of faces in the pictures are used. The assumption is that participants will not initially recognize any of the faces shown to them from the database. In total, 366 images were shown to participants across three phases (see Table 1 for a breakdown of phases and recognition classes within them). In the first phase, 162 images were shown which included 60 images that were repeated to be implicitly learned for the following phases, plus the 102 NR images. 102 images were shown in both phase two and phase three. 10 of the images in phase two were considered PUR images (learned in phase one) and the other 92 images were again NR images. In the third phase, another 102 images were shown with 10 PUR images as well as an additional 20 images for PAR, and the rest were NR images. Three phases were chosen to be able to separate the different recognition classes of images and to allow participants to implicitly learn faces in the first phase (day one of the experiment) that would be carried into the second and third phases (day two) as PUR images.

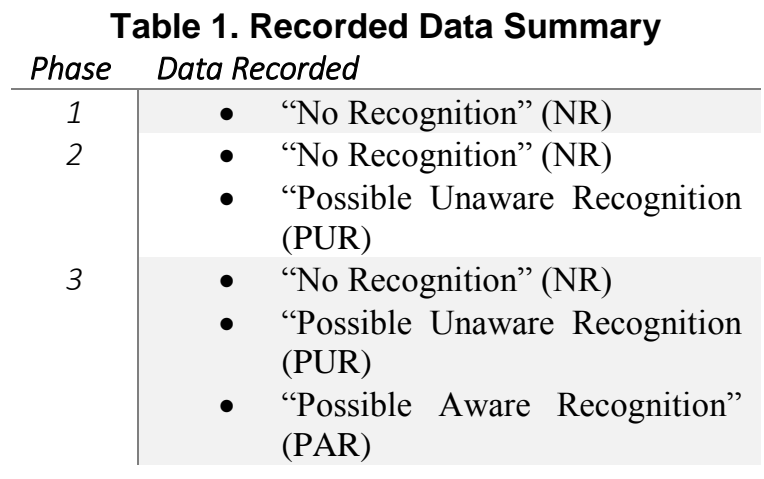

The experiment was conducted within a program written in Python by the research team. This software was designed to construct the subsets of faces that were shown to participants, organize the different sets into the three phases, display the images to the participants, and to send signals via emulated serial port to the EPOC headset to provide markers in the raw output describing when images were shown and what recognition class they belonged to. It was designed to be simple for the participants to use during the experiment and to minimize the amount of participant movement by removing any need to use the mouse or keyboard during the three phases as this may add artifacts to the signal output.

3.2.1. Phase One. In the first phase of the experiment, a number of images were shown to the participants of the study. They were tasked with simply considering the images shown to them. The goal of this phase was that the participants would not recognize any of the faces shown to them so that we could use the data gathered here as baseline "No Recognition" (NR) data for comparisons later on during the data analysis. In this phase the images that are to be shown as unaware images in later phases are shown to the participant a total of four times each to reinforce the implicit learning of the images.

3.2.2. Phase Two. In the second phase of the experiment, the same number of images were shown to the participant as in phase one, but a number of images were inserted into the list from phase one. The goal of this phase was to generate more NR data, but to also hopefully gather "Possible Unaware Recognition" (PUR) data when the participants view the images that were taken from the first phase. Ideally, the participants would view and implicitly learn the faces shown to them in phase one [28] [29] [30], and then elicit an unaware recognition of these images in phase two.

3.2.3. Phase Three. In the third and final phase of the experiment, participants were shown one of the images that was tagged as PUR from the second phase and asked to memorize the face. Once the participant felt comfortable enough with the face that they could remember it, they moved on and were again shown a number of images. The images in this phase consisted of a majority of NR images which they had not seen before, but also, spaced evenly throughout the images, there was the image of the face that they were asked to study at the beginning of the phase. Assuming the participant had memorized the face well enough to be able to recognize it, upon viewing that face, the participant would elicit a "Possible Aware Recognition" (PAR). This final classification of data allows us to now compare the EEG signals between the three classes (No Recognition, Possible Aware Recognition, and Possible Unaware Recognition). Since only a limited number of images could be taken from phase one and placed into phase two as PUR data images, more images were taken from phase one and placed into phase three to supplement the PUR data count. Each image shown to the participants in all three phases is shown for one second, with a one second blank screen in between before moving on to the next image. 
To ensure that data collected from viewing images belongs to the correct possible classification, we pre-defined images that were shown to the participant in the various stages based on what we are looking for an image to be associated with. For example, one of the images that exists in Phase One that is picked to be shown again in Phase Two as a PUR would be labelled as a PUR image on the backend. This allowed us to easily compare any classification with another by looking at the tag that was associated with it. This also helped us to form a more obvious baseline for each of the classifications as they may have traits such as average voltage level or peaks that occur at specific times across all images of the same class.

\subsection{Data Pre-Processing}

Prior to analysis, data was pre-processed to remove or fix any artifacts that were found in the data. EEGLAB was used for data analysis and preprocessing. This was chosen due to its large, mature development community and the libraries and functionality included. The first step taken to remove any artifacts caused by blinking or other disturbances was to run the data through a band-pass filter, filtering 0.1-15 Hz (as recommended by Bougrain et al [31]). Next, the filtered data was run through one of EEGLAB's ICA filters, which helps eliminate any remaining artifacts and smooth the waveform [32]. After filtering and early artifact reduction/removal via ICA filter, any sections of image data that exhibited amplitudes of +/- 100 microvolts (usually caused by movements of the participants such as coughing, head movements, blinking, etc.) were removed from consideration as the data for that image may corrupt any further analysis.

As a result of this data pre-processing, a number of images were removed from analysis per participant included in the study. An average of 16 and 18 images for Participant 1 and 2 respectively were removed per sensor. In future work this may cause issues if images such as the PUR images in phase two or PAR images in phase three become corrupt, thus leading to less data for meaningful analysis.

\section{Analysis and Results}

Analysis of the data collected for this preliminary study was limited to simple analysis of the voltage of collected signals. First, the absolute value of the data for every image in a class for each participant was averaged at each time interval to produce an "average signal". This allowed us to view and determine if a human-visible difference between the three classifications of recognition exists. The results of this signal averaging can be seen in the following four graphs (Figures 1, 2, 3 and 4), containing the averaged brain activity of the two subjects immediately following exposure to the presented images.

In figures 1 and 2, the average signals collected during seven unique ERPs are displayed for each of the three recognition types. ERPs were averaged for each participant individually to allow us to get a general sense of where each image type falls in terms of voltage for each participant and to determine if the difference between classifications of image can be determined by a human without the need for machine learning tools. For this experiment we segmented the data into the following ERPs: VEPS1, VEPS2, N170, P2, N200, P3N400, and N400F. These ERPs are just segments of time in which we expect the brain to react in a specific way, so each sensor's data can be segmented (14 sensors $\times 7$ ERPs). In each of the graphs, certain averaged signals cut off at certain points due to the length of the ERP they are associated with (some ERPs are shorter than others). In figures 3 and 4 , the signals for each recognition class is presented for the full length that an image was shown. The signals represented in these two figures are averaged among all samples of each of their respective classes. In all signal figures, green signals correspond to NR images, blue signals correspond to PUR images, and orange signals correspond to PAR images. All data represented in these graphs are left-aligned rather than being aligned to their actual ERP-defined offset (time after stimulus). This is done to prevent missing signal lines due to overlapping signals as certain ERPs exist within other ERPs, thus producing the same shape as another at certain times.

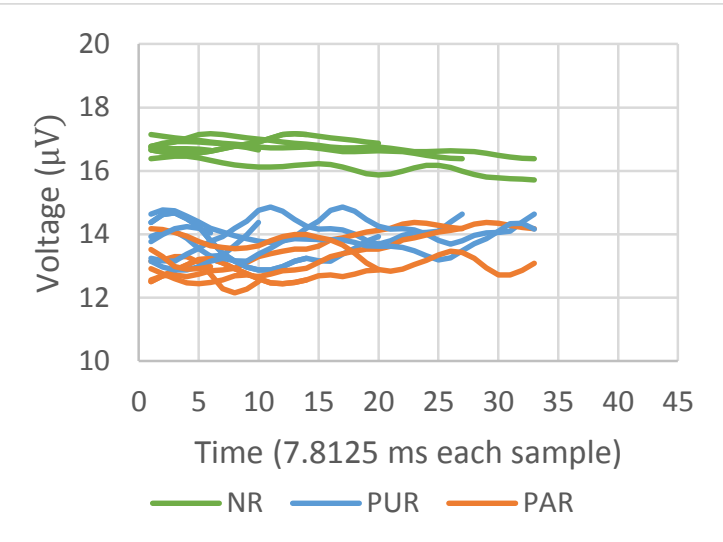

Figure 1 - Participant 1's averaged ERP signals 


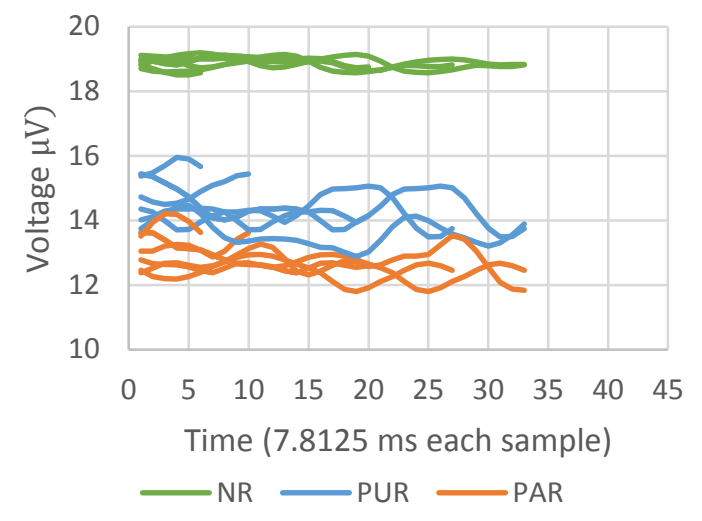

Figure 2 - Participant 2's averaged ERP signals

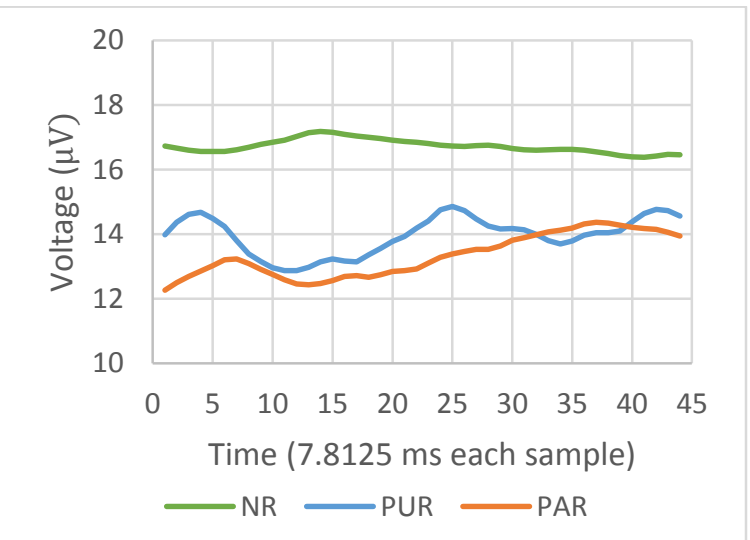

Figure 3 - Participant 1's averaged full-image signals

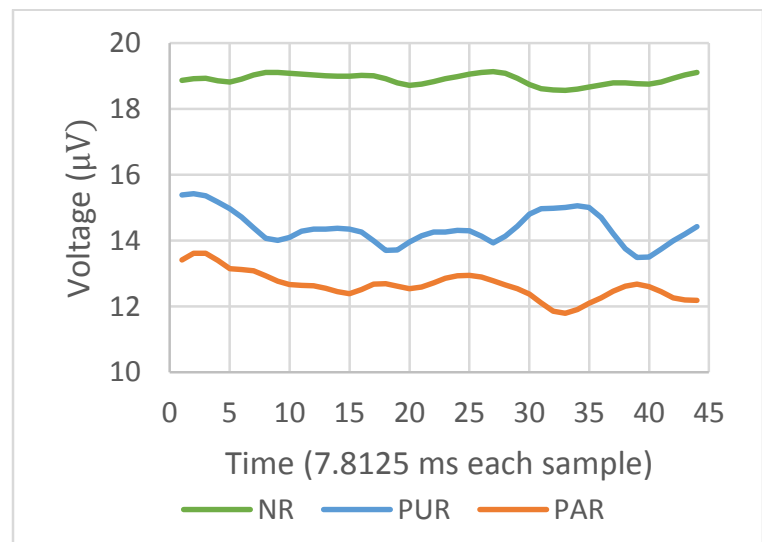

Figure 4 - Participant 2's averaged full-image signals

In the two trials conducted thus far, there is a clear distinction between NR, PUR, and PAR images, which supports the theory that consumer-grade BCI headsets can be used to identify possible unaware facial recognition. Unfortunately, being such a small sample size, we cannot make a statistical analysis on the results or develop more concrete conclusions of the results in this study. Studies are in the process of being conducted using more participants.

The differences between the three classes of recognition can be easily identified by the human eye without using any machine learning tools. The NR (green) images are generally of higher voltage levels and the PUR (blue) images appear slightly higher than the PAR (orange) images. The faces that are not recognized tend to be softer signals with less variance in voltage levels while the two classes that have recognition associated with them (PUR, PAR) tend to be more active in voltage changes. Considering that the aware recognitions (the faces that the participants were asked to specifically identify in phase three) share similar activity in the voltage levels as the unaware recognitions (phase two and three), this helps to reinforce that the brain is able to recognize these faces in a similar fashion to the aware recognitions, but at an unaware level. If reproducible in future work, these results may assist in applications which look to determine if an individual is recognized by another for example.

There are specific ERPs that lend themselves more to visual facial recognition than others, so future work will inspect these closer, but we have chosen to include the results of other well-known ERPs to see what insight we could gather on their function in relation to the ERPs associated with facial recognition and identification.

\section{Future Work}

For the purposes of this paper, the study outlined here is only a preliminary study to test the feasibility of the theory and processing behind the idea of classifying unaware recognitions. There is far more research to be done on the subject in both the medical field and in the Human-Computer Interaction field, leaving room for great advancements. One of the major areas that future work will continue with is the number of participants. For this experiment, participant numbers was kept low and involved only the research team surrounding this project, but future work would include a more general population, to allow for a more diverse data collection.

Another area to be investigated in future work is the left/right-handedness of the participants. The EPOC headset that was used for this experiment has 14 sensors (seven on each side of the head - left/right), so we can measure the certain areas of the brain that 
may be more responsive to viewing faces and reacting appropriately. While this preliminary experiment focuses purely on voltage levels, future work will differentiate voltage levels across the two halves of the brain to determine if a participant's dominant hand and areas of the brain play a role in classifying unaware facial recognitions.

While not performed on these initial results, we intend to use several additional methods of analysis in future trials. In addition to what was performed here, a variety of tools from the Scikit-Learn library for Python [33] will be used for preprocessing and classification. Initially, the feature set for all images that are analyzed consists of the voltage reading at each time interval $(128 \mathrm{~Hz}$, one sample per sensor every $7.8125 \mathrm{~ms}$ ).

This study was designed to take advantage of modern consumer-grade BCI headsets rather than the more expensive, elaborate, or even invasive lab/medical-grade devices. With the cost of such consumer-grade devices being appropriate for home or office use, additional applications are becoming available to be explored by a wider audience, which is what this study is directed towards. An area that this work could be taken in the future with regards to results is into a lab or medical study to delve further into the differences between aware and unaware recognitions.

\section{Conclusions}

While more participants are needed before any concrete conclusions can be made, preliminary results support the theory that consumer-grade BCI headsets can be used to identify unaware facial recognition. We explored the voltage differences that occur when the human brain is subjected to faces of varying states of recognition. From these limited results, we have found that the three states of recognition that we measured in the experiment (NR, PUR, and PAR) can be easily differentiated by taking the absolute values of signals after pre-processing. These results were obtained using a consumer-grade toy BCI headset rather than more expensive lab or medical grade headsets, thus lending credence to the idea that cheaper and more pervasive headsets found on the consumer marker can be used for recognition-based tasks. Finding such obvious differences in the voltages of these signals, we hope that scaling the experiment up to a larger sample size will produce a more statistically-sound result, along with a more in-depth analysis.

If future trials produce similar results, this knowledge could have several potential uses, including aiding workers in finding missing persons, other such recognition-based tasks, and the further inspiration for development and use of low-cost, consumer-grade toy BCIs for research purposes.

\section{Acknowledgements}

Portions of the research in this paper use the FERET database of facial images collected under the FERET program, sponsored by the DOD Counterdrug Technology Development Program Office. This work was supported in part by the Natural Sciences and Engineering Research Council of Canada.

\section{References}

[1] T. Shashibala and B. W. Gawali, "Brain Computer Interface Applications and Classification Techniques," International Journal of Engineering and Computer Science, vol. 5, no. 7, pp. 1726017267, 2016.

[2] EMOTIV Inc., "MindDrone," [Online]. Available: http://emotiv.com/product/minddrone/. [Accessed 01 06 2016].

[3] EMOTIV Inc., "Cortex Arcade," [Online]. Available: http://emotiv.com/product/cortex-arcade/. [Accessed 0106 2016].

[4] NeuroSky, "MyndPlay Sports: Bowling," [Online]. Available: http://store.neurosky.com/products/myndplay-sportsbowling. [Accessed 0106 2016].

[5] NeuroSky, "FlappyMind - iOS," [Online]. Available: http://store.neurosky.com/products/flappymind. [Accessed 0106 2016].

[6] B. Cahn and J. Polich, "Meditation States and Traits: EEG, ERP, and Neuroimaging Studies," Psychological Bulletin, vol. 132, no. 2, pp. 180-211, 2006.

[7] J. Chuang, H. Nguyen and C. Wang, "I Think, Therefore I Am: Usability and Security of Authentication Using Brainwaves," Lecture Notes in Computer Science, vol. 7862, pp. 1-16, 2013.

[8] E. Peck, B. Yuksel and A. Ottley, "Using fNIRS Brain Sensing to Evaluate Information Visualization Interfaces," in Proceedings of the SIGCHI Conference on Human Factors in Computing Systems - CHI '13, Paris, 2013.

[9] M. Vargas Martin, V. Cho and G. Aversano, "Detection of Subconscious Face Recognition Using Consumer-Grade Brain-Computer Interfaces," ACM Transactions on Applied Perception (TAP), vol. 14, no. 1, p. Article 7, 2016.

[10] S. Shalgi and L. Y. Deouell, "Is Any Awareness Necessary for an Ne?," Frontiers in Human Neuroscience, vol. 6, no. May, pp. 1-15, 2012. 
[11] A. Greenwald, T. Poehlman and E. Uhlmann, "Understanding and Using the Implicit Association Test," Journal of Personality and Social Psychology, vol. 97, no. 1, pp. 17-41, 2009.

[12] M. Seeck, C. Michel, N. Mainwaring, R. Cosgrove, H. Blume, J. Ives, T. Landis and D. Schomer, "Evidence for Rapid Face Recognition from Human Scalp and Intracranial Electrodes," Neuroreport, vol. 8, no. 12, pp. 2749-2754, 1997.

[13] N. George, B. Jemel, N. Fiori and B. Renault, "Face and Shape Repetition Effects in Humans: A SpatioTemporal ERP Study," Neuroreport, vol. 8, no. 6, pp. 1417-1423, 1997.

[14] B. J. Debruille, F. Guillem and R. Bernard, "ERPs and chronometry of face recognition: Following-up Seeck et al. and George et al.," Neuroreport, vol. 9, no. 15, pp. 3349-3353, 1998.

[15] M. Eimer, "Event-Related Brain Potentials Distinguish Processing Stages Involved in Face Perception and Recognition," Clinical Neurophysiology, vol. 111, no. 4, pp. 694-705, 2000.

[16] S. Bentin and L. Y. Deouell, "Structural Encoding and Identification in Face processing: ERP Evidence for Separate Mechanisms," Cognitive Neuropsychology, vol. 17, no. 1-3, pp. 35-55, 2000.

[17] S. Caharel, S. Poiroux, C. Bernard, F. Thibaut, R. Lalonde and M. Rebai, "ERPs Associated With Familiarity and Degree of Familiarity During Face Recognition," International Journal of Neuroscience, vol. 112, no. 12, p. 1499, 2002.

[18] S. Sternberg, "High-Speed Scanning in Human Memory," Science, vol. 153, no. 3736, pp. 652-654, 1966.

[19] E. V. Mnatsakanian and I. M. Tarkka, "Matching of Familiar Faces and Abstract Patterns: Behavioral and High-Resolution ERP Study," International Journal of Psychophysiology, vol. 47, no. 3, pp. 217-227, 2003.

[20] E. T. Solovey, F. Lalooses, A. Girouard, R. J. Jacob, K. Chauncey, D. Weaver, M. Parasi, M. Scheutz, A. Sassaroli, S. Fantini and P. Schermerhorn, "Sensing Cognitive Multitasking for a Brain-Based Adaptive User Interface," in Proceedings of the 2011 Annual Conference on Human Factors in Computing Systems - CHI '11, Vancouver, 2011.

[21] A. R. Harrivel, D. H. Weissman, D. C. Noll and S. J. Peltier, "Monitoring Attentional State with fNIRS," Frontiers in Human Neuroscience, vol. 7, no. December, p. 861, 2013.

[22] J. Lee and D. Tan, "Using a Low-Cost Electroencephalograph for Task Classification in HCI Research," in Proceedings of the 19th ACM Symposium on User Interface Software and Technology, Montreux, 2006.
[23] C. Brunner, A. Delorme and S. Makeig, "EEGLAB An Open Source MATLAB Toolbox for Electrophysiological Research," Biomedizinische Technik, vol. 58, no. 1, 2013.

[24] EMOTIV Inc., "Epoc+," [Online]. Available: http://emotiv.com/epoc/. [Accessed 0106 2016].

[25] F. W. Sharbrough, G. E. Chatrian, R. Lesser, T. W. Picton, h. Luders and M. Nuwer, "American Electroencephalographic Society Guidelines for Standard Electrode Position Nomenclature," Journal of Clinical Neurophysiology, vol. 8, no. 2, pp. 200202, 1991.

[26] P. J. Phillips, H. Wechsler, J. Huang and P. Rauss, "The FERET Database and Evaluation Procedure for Face Recognition Algorithms," Image and Vision Computing J, vol. 16, no. 5, pp. 295-306, 1998.

[27] P. J. Phillips, H. Moon, S. A. Rizvi and P. J. Rauss, "The FERET Evaluation Methodology for Face Recognition Algorithms," IEEE Trans. Pattern Analysis and Machine Intelligence, vol. 22, pp. 10901104, 2000.

[28] Y.-C. Tseng and C.-S. Ray Li, "Oculomotor Correlates of Context-Guided Learning in Visual Search," Perception \& Psychophysics, vol. 66, no. 8, pp. 1363-1378, 2004.

[29] M. M. Chun and Y. Jiang, "Contextual Cueing: Implicit Learning and Memory of Visual Context Guides Spatial Attention," Cognitive Psychology, vol. 36, no. 1, pp. 28-71, 1998.

[30] A. Goujon, A. Didierjean and S. Poulet, "The Emergence of Explicit Knowledge from Implicit Learning," Memory \& Cognition, vol. 42, no. 2, pp. 225-236, 2014.

[31] L. Bougrain, C. Saavedra and R. Ranta, "Finally, What is the Best Filter for P300 Detection?," TOBI Workshop III - Tools for Brain-Computer Interaction - 2012, vol. 2012, pp. 2-3, 2012.

[32] J. C. Liao and W. C. Fang, "An ICA-Based Automatic Eye Blink Artifact Eliminator for RealTime Multi-Channel EEG Applications," Digest of Technical Papers - IEEE International Conference on Consumer Electronics, vol. 1, no. 2, pp. 532-535, 2013.

[33] F. Pedregosa, G. Varoquaux, A. Gramfort, V. Michel, B. Thirion, O. Grisel, M. Blondel, P. Prettenhofer, R. Weiss, V. Dubourg, J. Vanderplas, A. Passos, D. Cournapeau, M. Brucher, M. Perrot and E. Duchesnay, "Scikit-learn: Machine Learning in Python," Journal of Machine Learning Research, vol. 12, pp. 2825-2830, 2012. 